\title{
Phase functions as solutions of integral equations
}

\author{
Margarita L. Shendeleva
}

\begin{abstract}
A phase function is an important characteristic of a scattering medium. A method to derive new analytic phase functions is proposed. The relation between a phase function and an angle-averaged single-scattering intensity, derived earlier [M. L. Shendeleva, J. Opt. Soc. Am. A 30, 2169 (2013)], is considered as an integral equation for a phase function. This equation is classified as an Abel integral equation of the first kind, whose solution is known. Two phase functions newly derived with this method are presented.
\end{abstract}

Keywords: Radiative transfer, Phase function, Successive scattering orders, Integral equation, Abel, Single scattering, Anisotropy, Henyey-Greenstein

\section{Background}

The radiative transfer equation (RTE), which models light propagation in scattering media, contains two unknown functions: the radiance and the scattering phase function. Usually, the phase function is modeled separately and then inserted into the RTE. A common approach to such modelling relies on the use of Mie theory [1], which models, using the Maxwell equations, the light scattering from a single spherical particle. Mie theory was also extended to particles of other shapes [2]. In aerosols and soft tissue, various sorts of averaging over particle size distributions are applied. After averaging, the Mie phase function appears to become much smoother and can be well approximated by a co-called analytic or parametric phase function.

A widely used one-parametric phase function is that derived by Henyey and Greenstein (HG) [3]:

$$
p^{H G}(\mu)=\frac{1-g^{2}}{4 \pi\left(1+g^{2}-2 g \mu\right)^{3 / 2}},
$$

where $g$ is a parameter in the range $[-1,1]$ and $\mu$ is the cosine of the scattering angle. The phase function is normalized such that

$$
2 \pi \int_{-1}^{1} p(\mu) d \mu=1
$$

A convenient property of the HG phase function is that the parameter $g$ is identically equal to the anisotropy (or asymmetry) factor $g^{a n i}$, defined as

$$
g^{a n i}=\frac{\int_{-1}^{1} \mu p(\mu) d \mu}{\int_{-1}^{1} p(\mu) d \mu} .
$$

Therefore, $g^{a n i}=g$ for the HG phase function, where $\left|g^{\text {ani }}\right| \leq 1$.

A generalization of the HG phase function was introduced by Reynolds and McCormick [4] as

$$
p^{R M}(\mu)=\frac{C}{\left(1+k^{2}-2 k \mu\right)^{\alpha+1}}
$$

where $C$ is the normalization factor,

$$
C=\frac{\alpha k\left(1-k^{2}\right)^{2 \alpha}}{\pi\left[(1+k)^{2 \alpha}-(1-k)^{2 \alpha}\right]},
$$

for $\alpha \neq 0$ and $C=k /\{2 \pi \ln [(1+k) /(1-k)]\}$ for $\alpha=0$. Here $\alpha$ and $k$ are real parameters, where $|k| \leq 1$. 
For $\alpha=1 / 2$ the Reynolds-McCormick function reduces to the HG phase function, and, for $\alpha=0$, it reduces to the ellipsoidal phase function

$$
p^{e l}(\mu)=\frac{k}{2 \pi \ln \left(\frac{1+k}{1-k}\right)\left(1+k^{2}-2 k \mu\right)},
$$

where $k$ is a real parameter related to the anisotropy factor as

$$
g^{a n i}=\frac{\left(1+k^{2}\right)}{2 k}-\frac{1}{\ln [(1+k) /(1-k)]},
$$

where $\left|g^{a n i}\right| \leq 1$.

More flexibility for modelling phase functions is obtained by combining two HG phase functions or a HG phase function with an isotropic phase function or with a delta function, yielding two-parameter phase functions [5] or three parameter phase functions [6].

As was pointed out by Selden [7], the main features of a phase function typically comprise a narrow forward lobe (corona), a broad diffuse background, and a narrow backscattering peak (glory). Various analytic phase functions that model these three major components were proposed by Cornette and Shanks [8], Liu [9], Draine [10] and many others (see the review of Sharma [11]). It should be noted that the influence of the choice of the analytic phase function in photon transport introduces errors in the determination of optical parameters that are difficult to evaluate [12].

Another approach to obtaining a phase function is based on understanding that the phase function can be found from the RTE itself, provided the radiance is known at some points. Such inverse problems for solving the RTE were considered by Zaneveld and Pak [13], Case [14], and McCormick [15]. The approach includes the decomposition of the phase function in a series of Legendre polynomials $P_{n}(\mu)$,

$$
p(\mu)=\frac{1}{4 \pi} \sum_{n=0}^{N} b_{n} P_{n}(\mu),
$$

where $N$ is finite. The radiance is also extended to a series of Legendre polynomials and, from the RTE, one eventually obtains a system of equations for unknown coefficients $b_{n}$.

In this paper, we apply an inverse procedure of a different kind. First, we expand the radiance in successive scattering orders and then exploit the relation between the first-order angle-averaged scattering intensity and the phase function. The first part of this problem was solved, using the successive order expansion developed by Paasschens [16], in Shendeleva [17]. Here we focus on the second part. Consider first a few examples illustrating the relation between the first-order scattering intensity and the phase function.
For the isotropic phase function,

$$
p^{i s o}=1 /(4 \pi),
$$

the first-order angle-averaged intensity generated by an instantaneous point source is [16]

$$
I_{1}^{i s o}=\frac{H(v t-r) e^{-v t / l_{s}}}{4 \pi v t r l_{s}} \ln \left(\frac{v t+r}{v t-r}\right)
$$

where $r$ is the distance from the point source, $t$ is the time from the moment of photon emission, $v$ is the speed of light in the medium, $l_{s}$ is the scattering length, and $\mathrm{H}(\mathrm{x})$ is the Heaviside step function that equals zero for $x<0$ and one for $x \geq 0$.

For the linear phase function

$$
p^{l i n}(\mu)=(1+3 g \mu) /(4 \pi),
$$

where parameter $|g| \leq 1 / 3$, the angle-averaged singlescattering intensity has been found as $[17,18]$

$$
I_{1}^{l i n}=\frac{e^{-v t / l_{s}} H(1-u)}{4 \pi r v t l_{s}}\left\{\left(1+3 g u^{2}\right) \ln \left(\frac{1+u}{1-u}\right)-6 g u\right\},
$$

where $u=r /(v t)$.

Generally, there is a one-to-one correspondence between a phase function and a first-order scattering intensity. For a given phase function, the first-order angle-averaged intensity (also called a single-scattering intensity) can be found from Eq. (21) of Shendeleva [17]. In this paper, we consider an inverse problem: Given the fist-order angle-averaged intensity, find the corresponding phase function. The integral equation for this purpose is derived in the next section. Fortunately, this equation happens to be an integral equation of the Abel type, whose solutions are known [19]. Sections 3 and 4 provide examples of the application of this equation.

\section{Derivation of the integral equation}

The time-dependent RTE for radiance $L(\vec{r}, t, \hat{s})$ is considered in the form

$$
\begin{aligned}
\frac{1}{v} \frac{\partial L(\vec{r}, t, \hat{s})}{\partial t} & +\hat{s} \nabla L(\vec{r}, t, \hat{s})+\left(\mu_{s}+\mu_{a}\right) L(\vec{r}, t, \hat{s}) \\
& =\mu_{s} \int_{4 \pi} p\left(\hat{s} \cdot \hat{s}^{\prime}\right) L\left(\vec{r}, t, \hat{s}^{\prime}\right) d \hat{s}^{\prime}+\frac{1}{v} S(\vec{r}, t),
\end{aligned}
$$

where $p\left(s \cdot \hat{s}^{\prime}\right)$ is a phase function, normalized such that $\int_{4 \pi} p\left(\hat{s} \cdot \hat{s}^{\prime}\right) d \hat{s}=1$, where $\hat{s}^{\prime}$ and $\hat{s}$ are directions before and after a scattering event, respectively, and $\mu_{s}$ and $\mu_{a}$ are the scattering and absorption coefficients, 
respectively, related to the scattering length $l_{s}=1 / \mu_{s}$ and to the absorption length $l_{a}=1 / \mu_{a}$. An instantaneous point source is represented by delta functions,

$$
S(\vec{r}, t)=\frac{N_{0} \hbar \omega v}{4 \pi} \delta(r) \delta(t)
$$

where $\hbar \omega$ is photon's energy, and $N_{0}$ is the number of photons emitted at $t=0$. Note that, in the following, we consider a non-absorbing case, since absorption enters into the solution through the exponential factor $\operatorname{Exp}\left(-\mu_{a} v t\right)(16)$.

The multiple collision approach uses the decomposition of the radiance in successive scattering orders

$$
L(\vec{r}, t, \hat{s})=\sum_{N=0}^{\infty} L_{N}(\vec{r}, t, \hat{s}),
$$

where the term with $N=0$ describes the unscattered radiance, the term with $N=1$ corresponds to the radiance scattered once, and so forth. Correspondingly, for the angle-averaged intensity, which is defined as

$$
I(\vec{r}, t)=\frac{1}{4 \pi} \int_{4 \pi} L(\vec{r}, t, \hat{s}) d \hat{s},
$$

we have the expansion

$$
I(\vec{r}, t)=\sum_{N}^{\infty} I_{N}(\vec{r}, t),
$$

where $I_{N}(\vec{r}, t)$ is an angle-averaged intensity of the N-s order defined as $I_{N}=\int_{4 \pi} L_{N}(\vec{r}, t, \hat{s}) d \hat{s} /(4 \pi)$.

For the first-order scattering, the relation between a phase function and first-order intensity is obtained as [17]

$$
I_{1}(r, t)=\frac{e^{-v t / l_{s}}}{l_{s}} \int_{-1}^{1} \frac{p(\mu) d \xi}{(v t)^{2}+r^{2}-2 v t r \xi},
$$

where $\mu$ is the cosine of the scattering angle $\theta$ and $\xi$ is the cosine of the angle $\alpha$ between the direction from the source to the observation point and the direction from the scattering point to the observation point (as shown in Fig. 1 of Shendeleva [17]). Using the notation $r=2 c$, $v t=2 a$, and $c / a=r /(v t)=u$, we can express cosines $\mu$ and $\xi$ as

$$
\begin{aligned}
& \xi=\frac{c-x}{a-u x}, \\
& \mu=\frac{2 c^{2}-a^{2}-u^{2} x^{2}}{a^{2}-u^{2} x^{2}},
\end{aligned}
$$

where $x$ is a coordinate along the $x$-axis in the range $[-a, a]$.

Changing the integration variable in Eq. (18) from $\xi$ to $\mu$, we transform Eq. (18) to

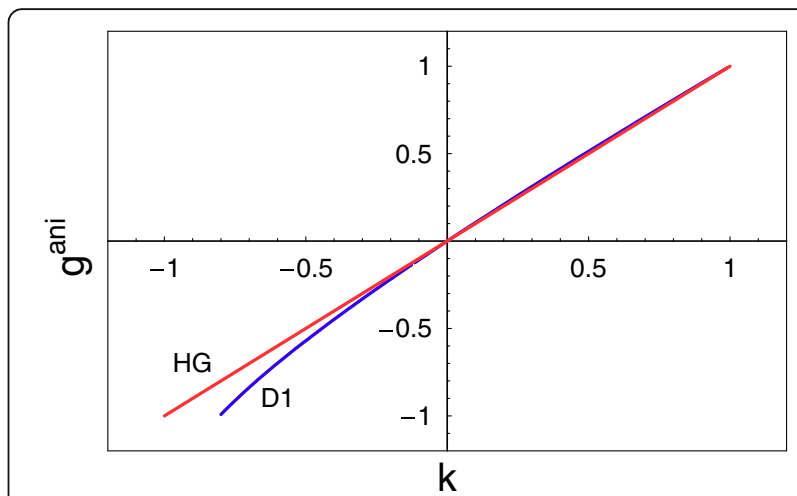

Fig. 1 The anisotropy factor $g^{\text {ani }}$ versus parameter $k$ for D1 phase function. For comparison, $g^{\text {ani }}=k$ for the HG phase function is shown

$$
I_{1}(r, t)=\frac{e^{-v t / l_{s}}}{v t r l_{s}} \int_{-1}^{X} \frac{p(\mu) d \mu}{\sqrt{1-\mu} \sqrt{X-\mu}}
$$

with $X=2 u^{2}-1$, where $X$ varies in the range $[-1,1]$.

Eq. (21) takes the form

$$
\int_{-1}^{X} \frac{p(\mu) d \mu}{\sqrt{1-\mu} \sqrt{X-\mu}}=F(X)
$$

and thus can be classified as an Abel integral equation of the first kind. The solution of this equation is known to be [19]

$$
p(\mu)=\frac{\sqrt{1-\mu}}{\pi} \int_{-1}^{\mu} \frac{F^{\prime}(\tau) d \tau}{\sqrt{\mu-\tau}}+\frac{F(-1) \sqrt{1-\mu}}{\sqrt{1+\mu}},
$$

where $F^{\prime}(\tau)$ means the derivative with respect to the argument. Note also the normalization conditions in Eq. (23). From the normalization of the phase function given by Eq. (2), one obtains the normalization for $F(\chi)$,

$$
\int_{-1}^{1} F(\tau) d \tau=\frac{1}{\pi}
$$

and therefore a single-scattering intensity is normalized as

$$
4 \pi \int_{0}^{v t} I_{1}(r, t) r^{2} d r=\frac{v t}{l_{s}} e^{-v t / l_{s}}
$$

which is consistent with the normalization obtained by Shendeleva [17].

\section{Examples of solutions}

Ellipsoidal phase function

To test the method, consider an ellipsoidal phase function given by Eq. (6). By direct substitution of this phase function in Eq. (18), one can find that the ellipsoidal phase function corresponds to the single-scattering intensity [17] 


$$
\begin{aligned}
I_{1}^{e l}(r, t) & =\frac{k e^{-\frac{v t}{l_{s}}}}{2 \pi r v t l_{s}(1-k) \ln \left(\frac{1+k}{1-k}\right) \sqrt{1+k^{2}-2 k \chi}} \\
& \ln \left(\frac{\sqrt{1+k^{2}-2 k \chi}+(1-k) \sqrt{(x+1) / 2}}{\sqrt{1+k^{2}-2 k \chi}-(1-k) \sqrt{(x+1) / 2}}\right),
\end{aligned}
$$

where $\chi=2 r^{2} /(v t)^{2}-1$.

Vice versa, considering the integral equation (22) with function

$$
\begin{aligned}
F^{e l}(x)= & \frac{k}{2 \pi(1-k) \ln \left(\frac{1+k}{1-k}\right) \sqrt{1+k^{2}-2 k \chi}} \\
& \ln \left(\frac{\sqrt{1+k^{2}-2 k \chi}+(1-k) \sqrt{(X+1) / 2}}{\sqrt{1+k^{2}-2 k \chi}-(1-k) \sqrt{(X+1) / 2}}\right),
\end{aligned}
$$

one obtains, after some manipulations, the ellipsoidal phase function (6).

The following considers the integral equations (22) with the right-hand side of the form

$$
F^{D a}(x)=\frac{\mathrm{K}}{\left(1+k^{2}-2 k \chi\right)^{a}} \ln \left(\frac{\sqrt{1+k^{2}-2 k \chi}+(1-k) \sqrt{(\chi+1) / 2}}{\sqrt{1+k^{2}-2 k \chi}-(1-k) \sqrt{(x+1) / 2}}\right),
$$

where $\mathrm{K}$ is the normalization factor that depends on parameter $k$. The superscript $D a$ indicates that expression $\left(1+k^{2}-2 k \chi\right)$ in the denominator enters in degree $a$. Consider the following two particular cases.

\section{Phase function D1}

In this case, $a=1$ and the right-hand side of Eq. (22) takes the form

$$
F^{D 1}(x)=\frac{\mathrm{K}}{1+k^{2}-2 k \chi} \ln \left(\frac{\sqrt{1+k^{2}-2 k \chi}+(1-k) \sqrt{(x+1) / 2}}{\sqrt{1+k^{2}-2 k \chi}-(1-k) \sqrt{(x+1) / 2}}\right),
$$

where $\mathrm{K}$ is found from the normalization condition (24) as

$$
\mathrm{K}=1 / \int_{-1}^{1} \frac{\pi}{1+k^{2}-2 k \chi} \ln \left(\frac{\sqrt{1+k^{2}-2 k x}+(1-k) \sqrt{\chi+1} / \sqrt{2}}{\sqrt{1+k^{2}-2 k \chi}-(1-k) \sqrt{\chi+1} / \sqrt{2}}\right) d x .
$$

Taking the derivative, one obtains

$$
\begin{gathered}
\left(F^{D 1}(\tau)\right)^{\prime}=\frac{2 k \mathrm{~K}}{\left(1+k^{2}-2 k \tau\right)^{2}} \ln \left(\frac{\sqrt{1+k^{2}-2 k \tau}+(1-k) \sqrt{\tau+1} / \sqrt{2}}{\sqrt{1+k^{2}-2 k \tau}-(1-k) \sqrt{\tau+1} / \sqrt{2}}\right) \\
+\frac{\sqrt{2}(1-k) \mathrm{K}}{\sqrt{1+\tau}(1-\tau)\left(1+k^{2}-2 k \tau\right)^{3 / 2}} .
\end{gathered}
$$

Thus, the solution for normalized phase function $p^{D 1}$ is found to be

$$
\begin{aligned}
p^{D 1}(\mu)= & \frac{\sqrt{1-\mu}}{\pi^{2}} \int_{-1}^{\mu}\left[\frac{2 k}{\left(1+k^{2}-2 k \tau\right)^{2}} \ln \left(\frac{\sqrt{1+k^{2}-2 k \tau}+(1-k) \sqrt{\tau+1} / \sqrt{2}}{\sqrt{1+k^{2}-2 k \tau}-(1-k) \sqrt{\tau+1} / \sqrt{2}}\right)\right. \\
& \left.+\frac{\sqrt{2}(1-k)}{\sqrt{1+\tau}(1-\tau)\left(1+k^{2}-2 k \tau\right)^{3 / 2}}\right] \frac{d \tau}{\sqrt{\mu-\tau}} \\
& / \int_{-1}^{1} \ln \left(\frac{\sqrt{1+k^{2}-2 k \tau}+(1-k) \sqrt{\tau+1} / \sqrt{2}}{\sqrt{1+k^{2}-2 k \tau}-(1-k) \sqrt{\tau+1} / \sqrt{2}}\right) \frac{d \tau}{\left(1+k^{2}-2 k \tau\right)} .
\end{aligned}
$$

It should be noted that $p^{D 1}(\mu)$ takes on negative values for $k<-0.8$; therefore, the range of parameter $k$ should be restricted to $-0.8<k<1$.

The anisotropy factor for this function is calculated as

$$
g^{a n i}=\frac{\int_{-1}^{1} \mu p(\mu) d \mu}{\int_{-1}^{1} p(\mu) d \mu}=\frac{\int_{-1}^{1}(1-\tau)(1+3 \tau) F^{\prime}(\tau) d \tau}{4 \int_{-1}^{1} F(\tau) d \tau} .
$$

The plot of the anisotropy factor versus parameter $k$ is shown in Fig. 1. Note that, for $-0.2<k<1$, the anisotropy factor closely follows the linear dependence $g^{a n i}=k$, which is characteristic of the HG phase function. In contrast to the HG phase function, the phase function D1 has a simple analytic form for the single-scattering angle-averaged intensity. It is shown in the Appendix that this fact can be used to approximate the singlescattering intensity for the HG phase function by the single-scattering intensity for the D1 phase function. A plot for the phase function $p^{D 1}(\mu)$ versus the scattering angle $\theta$ is shown in Fig. 2.

\section{Phase function D4}

Consider the integral equation (22) with the function

$$
F^{D 4}(x)=\frac{Q}{\left(1+k^{2}-2 k x\right)^{4}} \ln \left(\frac{\sqrt{1+k^{2}-2 k x}+(1-k) \sqrt{(x+1) / 2}}{\sqrt{1+k^{2}-2 k x}-(1-k) \sqrt{(x+1) / 2}}\right) .
$$

The notation D4 means that expression $\left(1+k^{2}-2 k x\right)$ enters the denominator in degree 4.

Here, $Q$ is the normalizing factor, which can be analytically calculated as follows. Changing back to the 


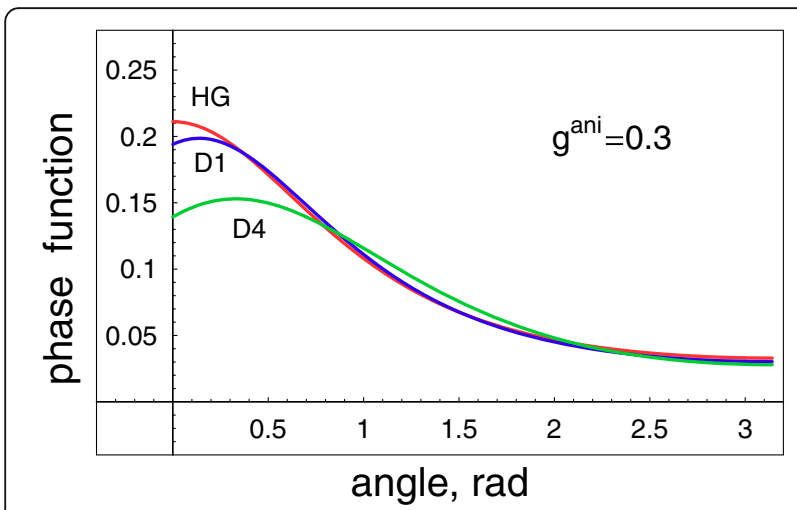

Fig. 2 Phase function $p(\cos \theta)$ versus the scattering angle $\theta$ for the three phase functions indicated in the figure

variable $u^{2}=(x+1) / 2$ in Eq. (34), one can write the normalization condition as

$$
\int_{0}^{1} \frac{\ln \left(\frac{\sqrt{(1+k)^{2}-4 k u^{2}}+(1-k) u}{\sqrt{(1+k)^{2}-4 k u^{2}}-(1-k) u}\right) u d u}{\left((1+k)^{2}-4 k u^{2}\right)^{4}}=\frac{1}{4 \pi} .
$$

Evaluating the integral with the use of Mathematica, we obtain

$$
Q=\frac{45(1-k)^{6}(1+k)^{6}}{4 \pi\left(45+60 k+158 k^{2}+60 k^{3}+45 k^{4}\right)}
$$

Therefore, the single-scattering intensity corresponding to $F^{D 4}(\chi)$ can be written in the form

$$
\begin{gathered}
I_{1}^{D 4}=\frac{45(1-k)^{6}(1+k)^{6} e^{-v t / l_{s}} H(1-u)}{4 \pi v t r l_{s}\left(45+60 k+158 k^{2}+60 k^{3}+45 k^{4}\right)\left[(1+k)^{2}-4 k u^{2}\right]^{4}} \\
\ln \left(\frac{\sqrt{(1+k)^{2}-4 k u^{2}}+(1-k) u}{\sqrt{(1+k)^{2}-4 k u^{2}}-(1-k) u}\right) .
\end{gathered}
$$

Calculating the derivative

$$
\begin{aligned}
& \left(F^{D 4}(\tau)\right)^{\prime}= \\
& =\frac{8 k Q}{\left(1+k^{2}-2 k \tau\right)^{5}} \ln \left(\frac{\sqrt{1+k^{2}-2 k \tau}+(1-k) \sqrt{\tau+1} / \sqrt{2}}{\sqrt{1+k^{2}-2 k \tau}-(1-k) \sqrt{\tau+1} / \sqrt{2}}\right) \\
& +\frac{\sqrt{2}(1-k) Q}{(1-\tau) \sqrt{1+\tau}\left(1+k^{2}-2 k \tau\right)^{9 / 2}},
\end{aligned}
$$

one obtains the phase function in the form

$$
\begin{array}{r}
p^{D 4}(\mu)=\frac{Q \sqrt{1-\mu}}{\pi} \int_{-1}^{\mu}\left[\frac{8 k}{\left(1+k^{2}-2 k \tau\right)^{5}}\right. \\
\ln \left(\frac{\sqrt{1+k^{2}-2 k \tau}+(1-k) \sqrt{(\tau+1) / 2}}{\sqrt{1+k^{2}-2 k \tau}-(1-k) \sqrt{(\tau+1) / 2}}\right) \\
\left.+\frac{\sqrt{2}(1-k)}{(1-\tau) \sqrt{1+\tau}\left(1+k^{2}-2 k \tau\right)^{9 / 2}}\right] \frac{d \tau}{\sqrt{\mu-\tau}},
\end{array}
$$

where $Q$ is given by Eq. (36).

Figure 3 shows the anisotropy factor $g^{a n i}$ versus parameter $k$. It can be seen that the curve $g^{a n i}(k)$ is closely approximated by the curve

$$
g^{a n i}=1-(1-k)^{2} /(1+k)^{2}
$$

for $0 \leq k \leq 1$. A polar plot for the phase function $p^{D 4}(\mu)$, and also for $p^{D 1}(\mu)$, is shown in Fig. 4. It should be noted that, for polar plots, it is convenient to make the change of variable $\tau+1=(1+\mu)\left(1-y^{2}\right)$, which transforms the integral in Eq. (39) to the integral with constant limits

$$
\begin{aligned}
p^{D 4}(\mu)=\frac{2 Q \sqrt{1-\mu^{2}}}{\pi} \int_{0}^{1}\left[\frac{8 k}{\left[(1+k)^{2}-2 k(1+\mu)\left(1-y^{2}\right)\right]^{5}}\right. \\
\\
\ln \left(\frac{\sqrt{(1+k)^{2}-2 k(1+\mu)\left(1-y^{2}\right)}+(1-k) \sqrt{\frac{(1+\mu)\left(1-y^{2}\right)}{2}}}{\sqrt{(1+k)^{2}-2 k(1+\mu)\left(1-y^{2}\right)}-(1-k) \sqrt{\frac{(1+\mu)\left(1-y^{2}\right)}{2}}}\right) \\
\left.+\frac{\sqrt{2}(1-k)}{\sqrt{1+\mu} \sqrt{1-y^{2}}\left[1-\mu+y^{2}(1+\mu)\right]\left((1+k)^{2}-2 k(1+\mu)\left(1-y^{2}\right)\right)^{9 / 2}}\right] d y .
\end{aligned}
$$

The use of the phase function $\mathrm{D} 4$ will be shown elsewhere.

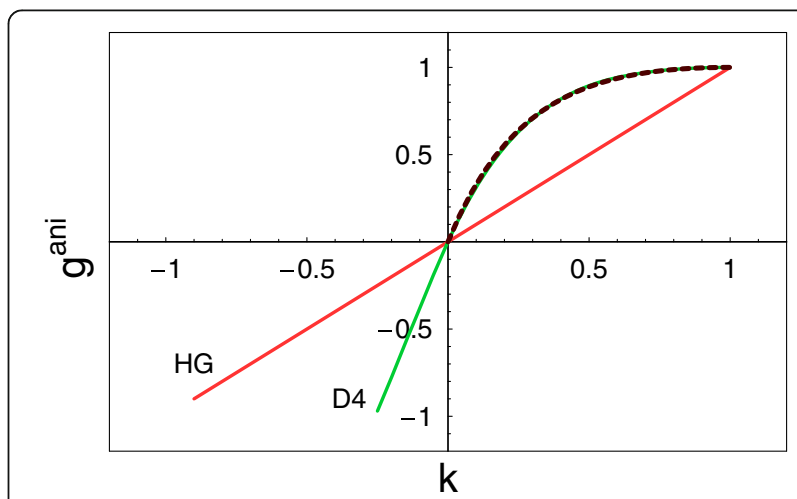

Fig. 3 The anisotropy factor $g^{\text {ani }}$ versus parameter $k$ for the D4 phase function and the HG phase function. The curve $g^{\text {ani }}=1-(1-k)^{2} /(1+$ $k)^{2}$ for $0<k<1$ is indicated by the dashed line 


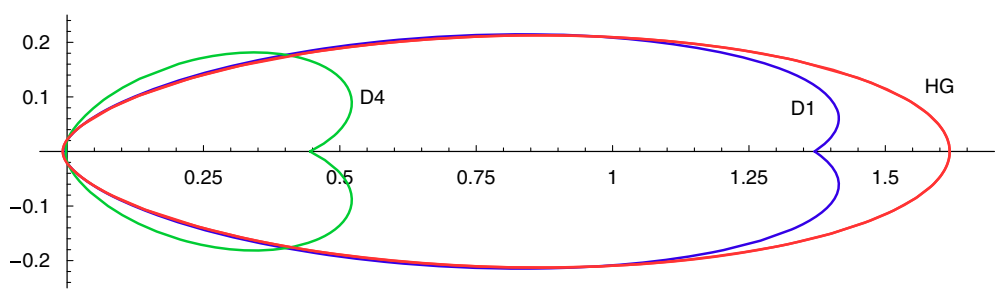

Fig. 4 Polar plot for the D1, D4 and HG phase functions for anisotropy factor $g^{a n i}=0.71$

\section{Conclusion}

We have derived an integral equation that relates a phase function and an angle-averaged intensity for the firstorder scattering, Eq. (21). The solution of this equation, classified as an Abel integral equation of the first kind, can be readily written down as given by Eq. (23). Then, we consider several examples of application of this equation. The first example is merely illustrative, since it concerns the ellipsoidal phase function for which the first-order intensity is known. This example is useful in the sense that it gives an idea of what the first-order intensity can look like. In the two subsequent examples, small modifications of the fist-order intensity allow us to derive two new oneparameter phase functions. The first one, denoted by D1, has the useful property that its anisotropy factor is practically identical to the parameter of the phase function (in the range $-0.2<k<1$ ). The same property is characteristic of the HG phase function. Although the function D1 is more complicated than the HG phase function, it has a very simple first-order scattering intensity. The second phase function, D4, has a simple algebraic relation (in the range $0<k<1$ ) between the anisotropy factor and the parameter of the phase function, as given by Eq. (40). These examples show that, through small modifications of the single-scattering intensity, one can derive new phase functions with useful properties.

\section{Appendix}

\section{Approximation of the single-scattering intensity for the} HG phase function

For the HG phase function, the single-scattering intensity is derived in the form $[17,20]$

$$
I_{1}^{H G}(r, t)=\frac{\left(1-g^{2}\right) e^{-v t / l_{s}}}{2 \pi l_{s}} \int_{0}^{1} \frac{\left(v^{2} t^{2}-r^{2} \lambda^{2}\right)^{1 / 2} d \lambda}{\left[\left(v^{2} t^{2}-r^{2} \lambda^{2}\right)(1-g)^{2}+4 g\left(v^{2} t^{2}-r^{2}\right)\right]^{3 / 2}} .
$$

Reilly and Warde [20] derived this expression for use in non-line-of-sight communications. For practical purposes, it would be useful to find an approximate analytical expression for this integral. Here, we propose approximating this expression by the first-order intensity found for the D1 phase function, since it has $k \approx g^{a n i}$ (for $-0.2<k<1$ ), similar to $g=g^{a n i}$ for HG. Thus, we obtain

$$
I_{1}^{H G}(r, t) \approx \frac{\mathrm{K}(g) e^{-v t / l_{s}}}{\operatorname{vtrl}_{s}\left[(1+g)^{2}-4 g u^{2}\right]} \ln \left(\frac{\sqrt{(1+g)^{2}-4 g u^{2}}+(1-g) u}{\sqrt{(1+g)^{2}-4 g u^{2}}-(1-g) u}\right),
$$

for $-0.2<g<1$. Here, $K(g)$, defined by Eq. (30), depends only on $g$ and, therefore, the dependence of $I_{1}^{H G}(r, t)$ on $r$ and $t$ is obtained in analytical form. Moreover, for $K(g)$, we find an approximation $K(g) \approx 0.05 g+0.08$ for $-0.2<g<1$. As a spinoff, we also obtain an approximation for the integral

$$
\begin{array}{r}
\int_{0}^{1} \frac{\left(1-u^{2} \lambda^{2}\right)^{1 / 2} d \lambda}{\left[\left(1-u^{2} \lambda^{2}\right)(1-g)^{2}+4 g\left(1-u^{2}\right)\right]^{3 / 2}} \approx \frac{2 \pi(0.05 g+0.08)}{u\left(1-g^{2}\right)\left[(1+g)^{2}-4 g u^{2}\right]} \\
\ln \left(\frac{\sqrt{(1+g)^{2}-4 g u^{2}}+(1-g) u}{\sqrt{(1+g)^{2}-4 g u^{2}}-(1-g) u}\right),
\end{array}
$$

where $-0.2<g<1$ and $0<u<1$. Denoting the left-hand side of the above equation by $\Upsilon_{L}$ and the right-hand side by $\Upsilon_{R}$, we calculate the relative error of this approximation as $\Delta=\left(\Upsilon_{R}-\Upsilon_{L}\right) / \Upsilon_{L}$. The relative error $\Delta$ for various parameters $g$ in shown in Fig. 5. It can be seen that the error is biggest for $u=0$. The error, which is bigger for large $g$, is decreasing in absolute value with increasing $u$ and, in the region $0.8<u<1$, it is less than $5 \%$.

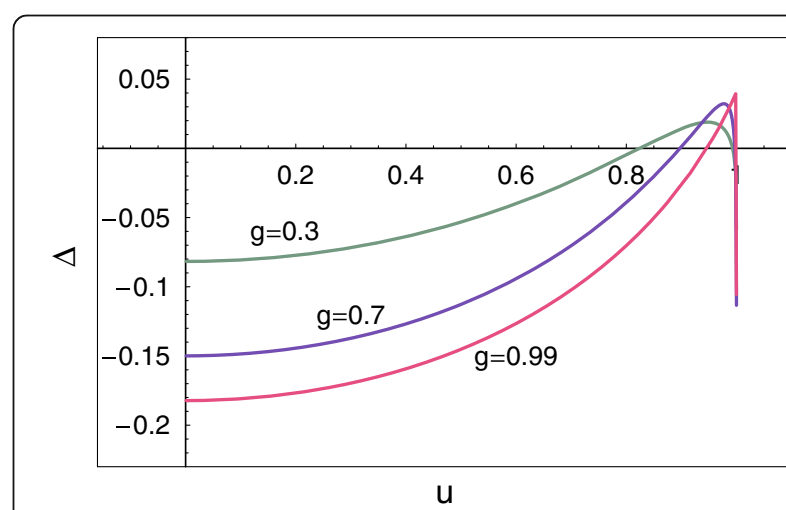

Fig. 5 Relative error $\Delta$ versus a dimensionless variable $u$ for the integral approximation given by Eq. (A3) 


\section{Competing interests}

The author declares that she has no competing interests.

\section{Publisher's Note}

Springer Nature remains neutral with regard to jurisdictional claims in published maps and institutional affiliations.

Received: 22 June 2016 Accepted: 8 March 2017

Published online: 24 March 2017

\section{References}

1. Mie, G.: Contributions to the optics of turbid media, particularly of colloidal metal solutions. [in German]. Annal. Phys. 25(N3), 377-445 (1908)

2. Bohren, C.F., Huffman, D.R.: Absorption and Scattering of Light by Small Particles. Wiley, New- York (1983)

3. Henyey, L.G., Greenstein, J.L.: Diffuse radiation in the galaxy. Astrophys. J. 93, 70-83 (1941)

4. Reynolds, L.O., McCormick, N.J.: Approximate two-parameter phase function for light scattering. J. Opt. Soc. Am. 70, 1206-1212 (1980)

5. Irvin, W.M.: Multiple scattering by large particles. Astrophys. J. 142, 1563-1575 (1965)

6. Kattawar, G.W.: A three-parameter analytic phase function for multiple scattering calculations. J. Quant. Spectrosc. Radiat. Transf. 15, 839-849 (1975)

7. Selden, A.C.: Attenuation and impulse response for multiple scattering of light in atmospheric clouds and aerosols. Appl. Opt. 45, 3144-3151 (2006)

8. Cornette, W.M., Shanks, J.G.: Physically reasonable analytic expression for the single-scattering phase function. Appl. Opt. 31, 3152-3160 (1992)

9. Liu, P.: A new phase function approximating to Mie scattering for radiative transport equations. Phys. Med. Biol. 39, 1025-1036 (1994)

10. Draine, B.T.: Scattering by interstellar dust grains. I. Opt. Ultraviolet Astrophys. J 598, 1017-1025 (1992)

11. Sharma, S.K.: A review of approximate analytic light-scattering phase functions. In: Kokhanovsky, A.A. (ed.) Light Scattering Reviews, vol. 9, pp. 53-100. Springer, Berlin (2015)

12. Selden, A.C.: Photon transport parameters of diffuse media with highly anisotropic scattering. Phys. Med. Biol. 49, 3017-3027 (2004)

13. Zaneveld, J.R.V., Pak, H.: Some aspects of the axially symmetric submarine daylight field. J. Geophys. Res. 77, 2677-2680 (1972)

14. Case, K.M.: Inverse problem in transport theory. Phys. Fluids 16 , 1607-1611 (1973)

15. McCormick, N.J.: Ocean optics phase-function inverse equations. Appl. Opt. 31, 4958-4961 (2002)

16. Paasschens, J.C.J.: Solution of time-dependent Boltzmann equation. Phys. Rev. E 56, 1135-1141 (1997)

17. Shendeleva, M.L.: Single-scattering solutions to radiative transfer in infinite turbid media. J. Opt. Soc. Am. A 30, 2169-2174 (2013)

18. Fomenko, V.N., Shvarts, F.M., Shvarts, M.A.: Exact description of photon migration in anisotropically scattering media. Phys. Rev. E 61, 1990-1995 (2000)

19. Bitsadze, A.V.: Integral Equations of First Kind. World Scientific, Singapore (1995)

20. Reilly, D.M., Warde, C.: Temporal characteristics of single-scatter radiation. J. Opt. Soc. Am. 69, 464-470 (1979)

\section{Submit your manuscript to a SpringerOpen ${ }^{\circ}$ journal and benefit from:}

- Convenient online submission

- Rigorous peer review

- Immediate publication on acceptance

Open access: articles freely available online

- High visibility within the field

- Retaining the copyright to your article

Submit your next manuscript at springeropen.com 